\title{
A cotransformation system of the unicellular red alga Cyanidioschyzon merolae with blasticidin S deaminase and chloramphenicol acetyltransferase selectable markers
}

Takayuki Fujiwara ${ }^{1,2^{*}+}$, Shunsuke Hirooka ${ }^{1+}$ and Shin-ya Miyagishima ${ }^{1,2^{*}}$

\begin{abstract}
Background: The unicellular red alga Cyanidioschyzon merolae exhibits a very simple cellular and genomic architecture. In addition, procedures for genetic modifications, such as gene targeting by homologous recombination and inducible/repressible gene expression, have been developed. However, only two markers for selecting transformants, uracil synthase (URA) and chloramphenicol acetyltransferase (CAT), are available in this alga. Therefore, manipulation of two or more different chromosomal loci in the same strain in C. merolae is limited.

Results: This study developed a nuclear targeting and transformant selection system using an antibiotics blasticidin $\mathrm{S}(\mathrm{BS})$ and the BS deaminase (BSD) selectable marker by homologous recombination in C. merolae. In addition, this study has succeeded in simultaneously modifying two different chromosomal loci by a single-step cotransformation based on the combination of BSD and CAT selectable markers. A C. merolae strain that expresses mitochondriontargeted mSCARLET (with the BSD marker) and mVENUS (with the CAT marker) from different chromosomal loci was generated with this procedure.

Conclusions: The newly developed BSD selectable marker enables an additional genetic modification to the already generated C. merolae transformants based on the URA or CAT system. Furthermore, the cotransformation system facilitates multiple genetic modifications. These methods and the simple nature of the $C$. merolae cellular and genomic architecture will facilitate studies on several phenomena common to photosynthetic eukaryotes.
\end{abstract}

Keywords: Cotransformation, Cyanidioschyzon merolae, Genetic modification, Photosynthetic eukaryote, Unicellular red alga

*Correspondence: tkfujiwara@nig.ac.jp; smiyagis@nig.ac.jp

${ }^{\dagger}$ Takayuki Fujiwara and Shunsuke Hirooka contributed equally to this work.

${ }^{2}$ Department of Genetics, Graduate University for Advanced Studies, SOKENDAl, Mishima, Shizuoka 411-8540, Japan

Full list of author information is available at the end of the article

\section{Background}

The unicellular red alga Cyanidioschyzon merolae is an emerging model organism for studies on phenomena common to photosynthetic eukaryotes $[1,2]$. The C. merolae nuclear and organellar genomes have been fully sequenced $[3,4]$. The nuclear genome composition is very simple with very little genetic redundancy (16.5 Mbp; 4775 protein-coding genes). The cellular 
organization is also very simple; it contains a single nucleus, mitochondrion, chloroplast, and a minimal number of other membranous organelles $[1,2]$. These organelles divide in a cell cycle-dependent manner, and cell cycle progression can be highly synchronized to a $12 \mathrm{~h}$ light $/ 12 \mathrm{~h}$ dark cycle $[1,2,5]$. In addition, a genetic modification procedure via homologous recombination has been established. Based on this procedure, various genetic techniques, such as gene knockout [6], gene knock-in [7], stable expression of a transgene without any silencing activity [8], and inducible/repressible expression of an endogenous gene or transgene [9-11], have been developed. The combination of these genomic and cytological features and molecular genetic techniques in C. merolae has facilitated analyses on a variety of cellular phenomena in photosynthetic eukaryotes, such as cell cycle progression [7, 12], organelle division and inheritance [13-18], circadian rhythms [19], metabolism [20-22], photosynthetic apparatus [23-26], splicing [27], and epigenetics [28].

To manipulate the $C$. merolae nuclear genome, two kinds of selectable markers, uracil synthase $(U R A)$ gene $[6,8]$ and chloramphenicol (CP) acetyltransferase (CAT) gene $[29,30]$, have been developed. When multiple modifications on different chromosomal loci are required in a single strain, they can be achieved by a two-step transformation with $U R A$ and CAT markers [30] or by a marker recycling system, in which the URA marker is designed to be eliminated after the selection of transformants via intrachromosomal homologous recombination [31]. However, these sequential genetic modification methods are practically time-consuming (it takes $\geq 4$ weeks for one round of genetic modification from transformation to obtain a liquid culture of a transformant before another round of genetic modification). To apply a $U R A$ selectable marker for genetic modification, the parental strain should be a uracil auxotrophic strain [32, 33]. Thus, a transformant generated using the CAT marker and wild-type (WT) cells as a parental strain can no longer be genetically modified. To overcome these limitations, this study developed a new drug-resistant selection system of C. merolae transformants that can work solely or in combination with the $C A T$ marker system.

Blasticidin S (BS) was initially identified as an antibiotic inhibiting growth of the fungus Piricularia oryzae, a cause of rice plant disease [34]. The antibiotic is a nucleoside analogue (molecular wight 422) originally isolated from the bacterium Streptomyces griseochromogenes [34]. BS binds to the peptidyl transferase center of the large ribosomal subunit (50S and 60S in bacteria and eukaryotes, respectively) and thereby inhibits protein synthesis in eukaryotes and prokaryotes by interfering with elongation and termination step [35, 36].
BS deaminase (BSD) detoxifies BS by catalyzing the deamination of a cytosine moiety in BS [37-39]. BSD belongs to the deaminase superfamily that are involved in nucleotide and ADP-ribose metabolism, and distributed through in eukaryotes, bacteria, and phases [37]. The $B S D$ gene was found only in the phyla Firmicutes and Actinobacteria in bacteria and fungi in eukaryotes [37], and cloned from the bacterium Bacillus cereus [40] and the fungi Aspergillus terreus [41] and has been used as a marker for selecting genetically manipulated strains in a variety of organisms, such as mammals [42], plants [40], yeasts [43], and the green alga C. reinhardtii [44], which do not possess $B S D$ gene.

This study developed a transformant selection system using BS and BSD of $A$. terreus in C. merolae. In addition, the $B S D$ selectable marker can be used in combination with the already developed CAT marker system to simultaneously modify two different chromosomal loci by a single-step cotransformation. These procedures will facilitate studies using multiple genetic modifications in C. merolae.

\section{Results and discussion Nuclear transformation system with the BSD selectable marker in C. merolae}

To validate the application of $\mathrm{BS}$ and $B S D$ to the nuclear transformation of $C$. merolae, a selectable marker consisting of the promoter of C. merolae $C A B$ gene (chlorophyll $\mathrm{a} / \mathrm{b}$ binding protein; CMN234C), BSD orf of $A$. terreus (UniProtKB/Swiss-Prot ID of the amino acid sequence, P0C2P0.1; the nucleotide sequence was codon-optimized to the $C$. merolae nuclear genome), and $3^{\prime}-u t r$ of the $C$. merolae APX gene (ascorbate peroxidase; CMM158C) was constructed (Fig. 1a). In addition, an expression cassette of a transgene consisting of the promoter of the $C$. merolae FBA gene (fructose-1,6-biphosphate aldolase; CMM158C), sequence encoding mitochondrion-targeted peptide [N-terminal 77 amino acids [45]; of C. merolae $\mathrm{EFTu}$ (elongation factor thermo unstable; CMS502C)], mSCARLET (a monomeric red fluorescent protein) orf, and $3^{\prime}$-utr of the C. merolae $\beta$-tubulin gene (CMN263C) was constructed to fluorescently label the mitochondrion of transformant cells (Fig. 1a). The $C A B$ and FBA promoters were chosen because a previous RNA-seq analysis showed that the mRNA abundance of these genes was relatively high in logarithmically growing $C$. merolae cells in the light [10]. The BSD selectable marker and the mitochondrion-targeted mSCARLET (MITMSCARLET) expression cassette were combined and sandwiched between $C$. merolae chromosomal sequences around CMD184C and CMD185C loci. This was done to integrate the BSD selectable marker and MITmSCARLET expression cassette into a chromosomal neutral locus 


\section{a}

\section{$B S D$}

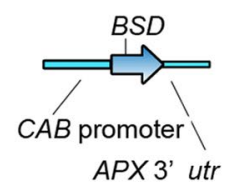

MITISSCARLET

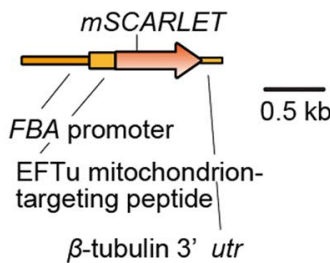

B-tubulin 3' utr
C

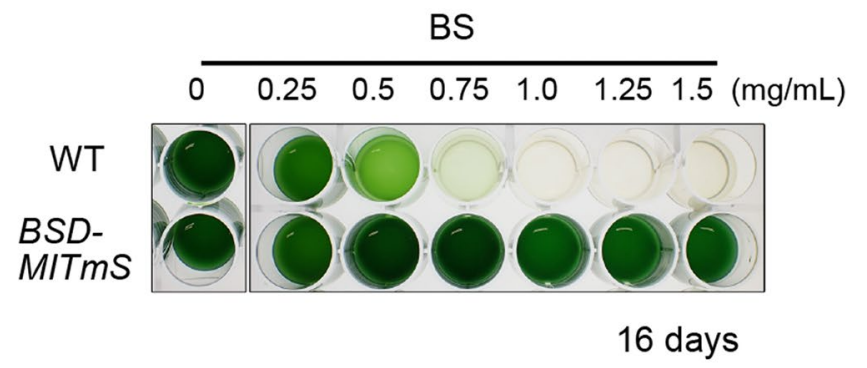

d

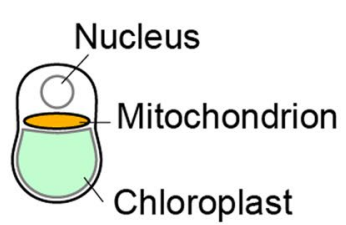

Fig. 1 C. merolae transformation system using BS and BSD selectable marker. (a) Schematic diagram of the BSD selectable marker and MITmSCARLET expression cassette. To constitutively express BSD as a selectable marker in C. merolae, BSD orf of A. terreus was codon-optimized to the C. merolae nuclear genome and conjugated with the C. merolae CAB promoter and APX $3^{\prime}-u$ tr. To constitutively express MITmSCARLET (mSCARLET connected to the mitochondrion-targeted sequence of $C$. merolae EFTu) as a transgene, MITmSCARLET orf was conjugated with the C. merolae FBA promoter and $\beta$-tubulin 3'-utr. (b) Schematic diagram of the insertion of the BSD selectable marker and MITmSCARLET expression cassette into an intergenic region between CMD184C and CMD185C by homologous recombination. The first line indicates the introduced linear DNA, the second line indicates the genomic structure of the WT strain, and the third line indicates the expected genomic structure of the transformant (BSD-MITmS). (c) Selection of BS-resistant transformants in a liquid medium. After PEG-mediated transformation, cells were recovered in a drug-free MA2 liquid medium for 2 days in the light. Cells were transferred to the MA2 liquid medium supplemented with a series of BS concentrations and incubated for 16 days in the light in a 24-well plate. WT cells were also cultured as a negative control. (d) Fluorescent micrographs showing mSCARLET fluorescence detected in BS-resistant transformants in the medium supplemented with $1 \mathrm{mg} / \mathrm{mL}$ BS. Cells were observed 14 days after inoculation of cells into the medium. A schematic diagram of a C. merolae cell is also shown. The cell has a single disk-shaped mitochondrion and a single cup-shaped chloroplast. PC, phase-contrast; orange, fluorescence of mSCARLET; red, autofluorescence of the chloroplast. Bar, $5 \mu \mathrm{m}$

between CMD184C and CMD185C [8] by homologous recombination (Fig. 1b). The resultant C. merolae transformant was named BSD-MITmS.

After introducing DNA to C. merolae WT cells by the polyethylene glycol (PEG)-mediated method $[2,46]$, cells were recovered for 2 days in an inorganic MA2 liquid medium without any drug in the light and transferred to the liquid medium with BS. To determine an appropriate BS concentration, WT cells and the BSD-MITmS transformant were cultured with a series of BS concentrations $(0,0.25,0.5,0.75,1.0,1.25$, and $1.5 \mathrm{mg} / \mathrm{mL})$ in the light for 16 days (Fig. 1c). As a result, WT cells died with BS in a dose-dependent manner, whereas the transformant grew in MA2 liquid medium supplemented with 1.0, 1.25, and $1.5 \mathrm{mg} / \mathrm{mL}$ BS (Fig. 1c). Fluorescence microscopy showed that mSCARLET fluorescence in the mitochondrion in most algal cells in the medium with $1.0 \mathrm{mg} / \mathrm{mL} \mathrm{BS}$, indicating that the transgene (MITmSCARLET) was successfully transformed into cells (Fig. 1d). However, some cells in the medium with $1.0 \mathrm{mg} / \mathrm{mL}$ BS lacked mSCARLET, suggesting that an unexpected integration of the $B S D$ marker, such as truncation of the MITmSCARLET expression cassette, likely occurred in these nonfluorescent cells. Thus, we then tried to isolate clones with an expected integration of the selectable marker and the transgene.

\section{Selection and isolation of BS-resistant transformant clones} on a gellan gum-solidified medium

A gellan gum-solidified MA2 medium supplemented with BS was prepared. The medium was covered with a cornstarch bed (Fig. 2a) to facilitate the colony formation of C. merolae [6, 8]. After the PEG-mediated introduction of DNA into cells, cells were recovered in MA2 liquid medium in the light for 2 days. Then, $50 \mu \mathrm{L}$ of the culture, which was diluted to give a concentration of $\mathrm{OD}_{750}=0.01$, were inoculated onto the cornstarch bed with $\mathrm{BS}$ and incubated in a $\mathrm{CO}_{2}(3 \%)$ incubator at $42^{\circ} \mathrm{C}$ in 


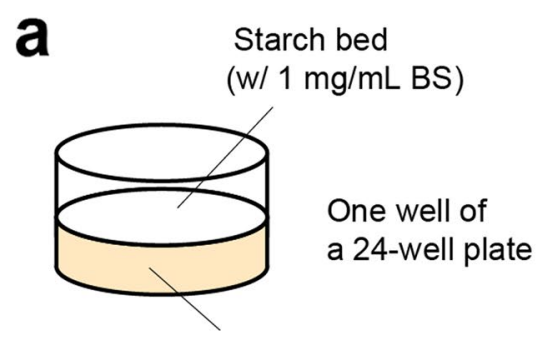

MA2 solidified with gellan gum (w/ $1 \mathrm{mg} / \mathrm{mL}$ BS)

b

BS $1 \mathrm{mg} / \mathrm{mL}$

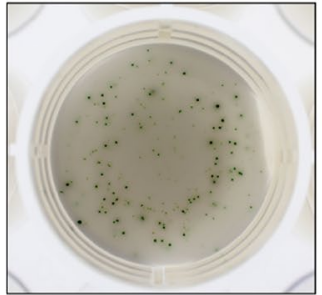

14 days

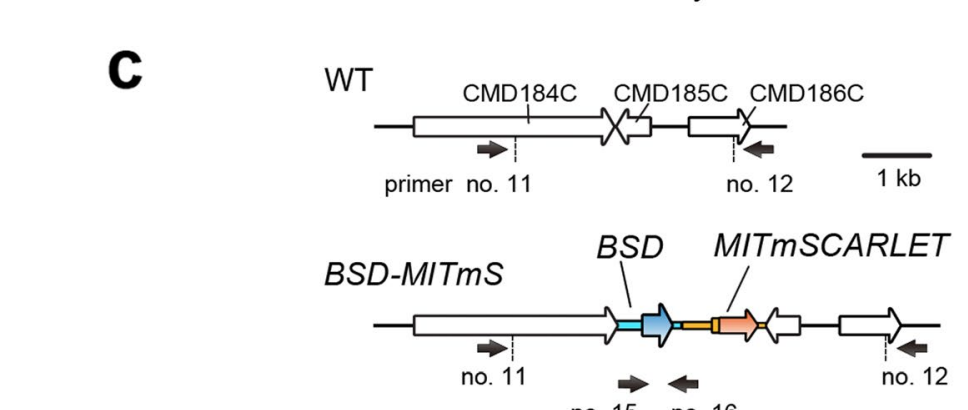

no. 15 no. 16
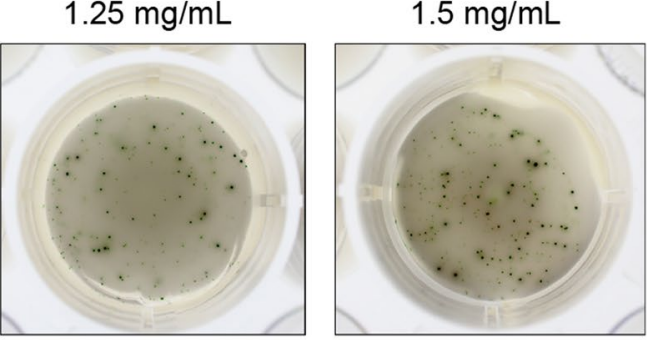
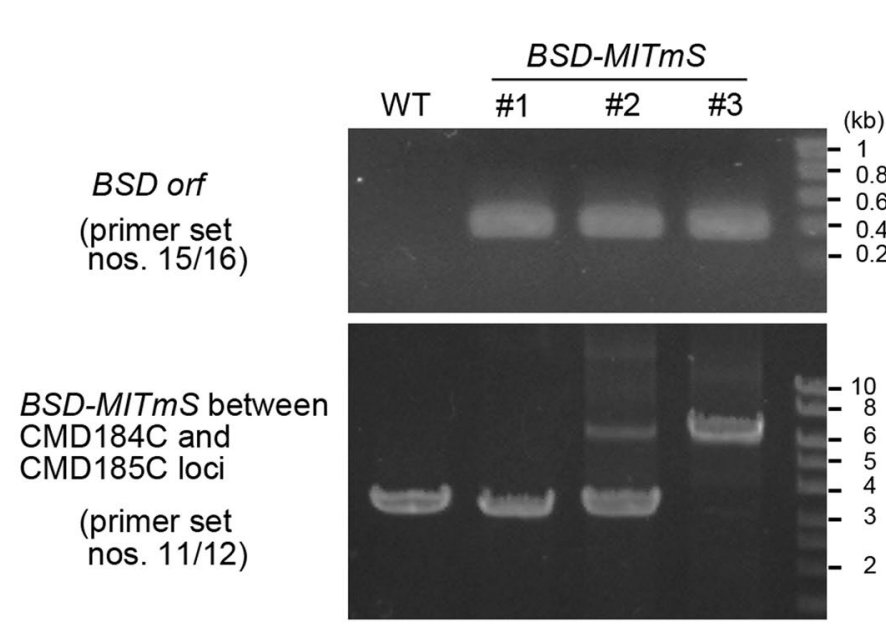

\#1. off-target

\#2. mixture of \#1 and \#3

\#3. on-target

$\begin{array}{llll}11 & 6 & 4 & \text { transformant colonies }\end{array}$

Fig. 2 Selection and isolation of BS-resistant transformant clones on a gellan gum-solidified medium supplemented with BS. (a) Schematic diagram of the MA2 gellan gum plate supplemented with $1 \mathrm{mg} / \mathrm{mL}$ BS. MA2 gellan gum supplemented with $1 \mathrm{mg} / \mathrm{mL}$ BS was solidified in one well of a 24-well plate. The medium was covered with a cornstarch bed to facilitate the colony formation of $C$. merolae. (b) Colonies of BS-resistant clones on MA2 gellan gum plate supplemented with 1.0, 1.25 and $1.5 \mathrm{mg} / \mathrm{mL}$ BS. After PEG-mediated transformation, cells were recovered in a drug-free MA2 liquid medium for 2 days in the light. Cells were spread on MA2 gellan gum plate supplemented with BS and incubated for 14 days in the light. (c) Colony PCR analyses of BS-resistant clones. The positions of the primers are indicated as arrows below the chromosomal structure of the WT and BSD-MITmS transformant. For detection of the BSD marker insertion either by off-target and on-target insertion (the primer set, nos. 15/16), the predicted size of the PCR product was $0.4 \mathrm{~kb}$. In the PCR amplifying CMD184C-CMD186C loci (the primer set, nos. 11/12), the predicted size of the PCR product of on-target insertion of the BSD-MITmS construct was $6.3 \mathrm{~kb}$ and the size for off-target insertion or the WT chromosome was $3.3 \mathrm{~kb}$. The exact positions and sequences of the primers are indicated in Supplementary Table S1. Full length unprocessed gel image of Fig. $2 \mathrm{c}$ is shown in Supplementary Fig. S1a. 
the light. After incubation for 14days, colonies appeared on the $\mathrm{BS}$ gellan gum plate (Fig. $2 \mathrm{~b}$ ).

To verify the targeted insertion of the $B S D$ marker and MITmSCARLET into the intergenic region between CMD184C and CMD185C, polymerase chain reaction (PCR) analyses of the colonies were performed. Typical examples of electrophoretic patterns of the PCR products are shown in Fig. 2c and Supplementary Fig. S1a. The PCR analyses showed that the $B S D$ marker was integrated into the genome of all transformant colonies examined (21/21 colonies; \#1-\#3 on the upper gel in Fig. 2c and Supplementary Fig. 1a). In contrast, the PCR analysis amplifying the CMD184C-CMD186C loci showed three patterns depending on the transformant colony; a single band showing off-target insertion of the transgene (11/21 colonies; \#1 on the lower gel in Fig. 2c and Supplementary Fig. S1a), two bands showing on-target and off-target insertions of the transgene $(6 / 21$ colonies; \#2 on the lower gel in Fig. 2c and Supplementary Fig. S1a), and a single band showing on-target insertion of the transgene (4/21 colonies; \#3 on the lower gel in Fig. 2c and Supplementary Fig. S1a).

\section{Simultaneous modification of two different chromosomal loci by a single-step cotransformation with $B S D$ and $C A T$ selectable markers in C. merolae}

To develop an efficient and multiple genetic modification system in C. merolae, two different chromosomal loci were modified simultaneously using both $B S D$ and $C A T$ selectable markers. In addition to BSD-MITmS (Figs. 1b and 3a), another construct consisting of the CAT selectable marker and mVENUS transgene $(C A T-m V)$ was prepared and designed to be integrated into an upstream region of CMK046C locus as another chromosomal neutral locus [10] (Fig. 3a). $B S D-M I T m S$ and CAT- $m V$ were simultaneously introduced to $C$. merolae WT cells by the PEG-mediated method (Fig. 3a). After recovery in drug-free MA2 liquid medium for 2 days, transformants were selected in MA2 liquid medium supplemented with both BS (1 mg/ $\mathrm{mL})$ and $\mathrm{CP}(0.2 \mathrm{mg} / \mathrm{mL})$ rather than gellan gum-solidified medium. This was because, in a previous study, CP-resistant transformants could grow in MA2 liquid medium supplemented with $\mathrm{CP}$ but were not able to form colonies on MA2 gellan gum plate supplemented with CP [30]. After incubation in MA2 liquid medium supplemented with BS and CP in the light for 14 days, WT died, whereas the transformant survived (Fig. 3b). The transformant culture was spread and incubated on a drug-free MA2 gellan gum plate to generate single colonies. PCR analyses of the colonies showed that the $B S D$ marker was integrated into the genome of all transformant colonies examined (21/21 colonies; \#1-\#3 on the upper gel in Fig. 3c and Supplementary Fig. S1b). $B S D$-MITmSCARLET was inserted into the intergenic region between CMD184C and CMD185C by on-target insertion in some colonies $(7 / 21$ colonies; \#3 on the middle gel in Fig. 3c and Supplementary Fig. S1b) and the $C A T-m V$ vector was inserted into the intergenic region of CMK046C upstream by on-target insertion in all colonies examined (21/21 colonies; \#1, \#2, and \#3 on the lower gel in Fig. 3c and Supplementary Fig. S1b). In the transformant clones, in which both transgenes were inserted by on-target insertion (\#3), fluorescence microscopy showed that the mitochondrion was labeled with mSCARLET fluorescence, and the cytosol

\footnotetext{
(See figure on next page.)

Fig. 3 Simultaneous modification of two different chromosomal loci by a single-step cotransformation with BSD and CAT selectable markers in C. merolae. (a) Schematic diagram of the insertion of the mVENUS transgene with the CAT selectable marker and that of the MITmSCARLET transgene with the BSD selectable marker into two different chromosomal loci by homologous recombination. (1) Schematic diagram of the insertion of the MITmSCARLET transgene with the BSD selectable marker as in Fig. 1a. (2) Schematic diagram of the mVENUS expression cassette and the CAT selectable marker. To constitutively express mVENUS, mVENUS orf was conjugated with the C. merolae CPCC promoter and ubiquitin $3^{\prime}$-utr. To constitutively express the CAT selectable marker, CAT orf was conjugated with the C. merolae APCC promoter and $\beta$-tubulin $3^{\prime}$-utr. (3) Schematic diagram of the insertion of the mVENUS transgene with the CAT selectable marker into a chromosomal neutral locus. The mVENUS transgene was connected with the CAT selectable marker and integrated into the chromosomal region at CMK046C upstream. The first line indicates the introduced linear DNA, the second line indicates the genomic structure of the WT chromosome, and the third line indicates the expected genomic structure of the transformant (CAT-mV). (b) Selection of BS- and CP-resistant transformants in MA2 liquid medium supplemented with BS and CP. After PEG-mediated transformation of the two constructs, cells were recovered in a drug-free MA2 liquid medium for 2 days in the light. Cells were transferred to MA2 liquid medium supplemented with $1 \mathrm{mg} / \mathrm{mL} \mathrm{BS}$ and $0.2 \mathrm{mg} / \mathrm{mL} C P$ and incubated for 14 days in the light in a 24-well plate. WT cells were also cultured as a negative control. (c) Colony PCR analyses of BS- and CP-resistant clones. The primer positions are indicated in (a). The transformants in the liquid culture above were spread onto a drug-free MA2 gellan gum plate to generate colonies of transformant clones before the PCR analyses. For detection of the BSD marker insertion either by off-target and on-target insertion (the primer set, nos. 15/16), the predicted size of the PCR product was $0.4 \mathrm{~kb}$. In the PCR amplifying CMD184C-CMD186C loci (the primer set, nos. 11/12), the predicted size of the PCR product of on-target insertion of the BSD-MITmS construct was $6.3 \mathrm{~kb}$ and the size for off-target insertion or the WT chromosome was $3.3 \mathrm{~kb}$. In the PCR amplifying the CMK046C locus and its upstream (the primer set, nos. 13/14), the predicted size of the PCR product of on-target insertion of the CAT-mV construct was $7.5 \mathrm{~kb}$ and the size for off-target insertion or the WT chromosome was $4.3 \mathrm{~kb}$. The exact positions and sequences of the primers are indicated in Supplementary Table S1. Full length unprocessed gel images of Fig. 3c are shown in Supplementary Fig. S1 b. (d) Fluorescent micrographs showing mSCARLET fluorescence in the mitochondrion (MITmSCARLET, orange) and mVENUS (green) fluorescence in the cytosol in the BSD-MITmS/CAT-mV transformant clone. PC, phase-contrast; red, autofluorescence of the chloroplast. Bar, $5 \mu \mathrm{m}$
} 


\section{a}

(1)

Between CMD184C and CMD185C loci

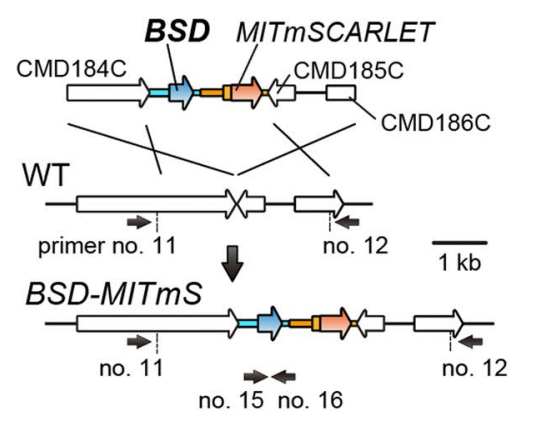

\section{(2)}

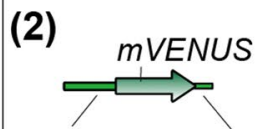

CPCC promoter ubiquitin 3' utr

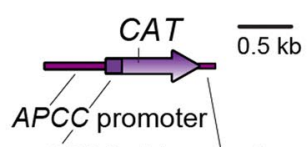

APCC chloroplasttargeting peptide

$$
\beta \text {-tubulin 3' utr }
$$

(3) Upstrem of CMK046C locus

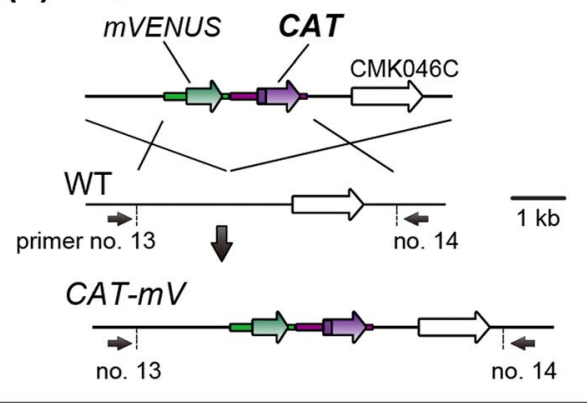

b

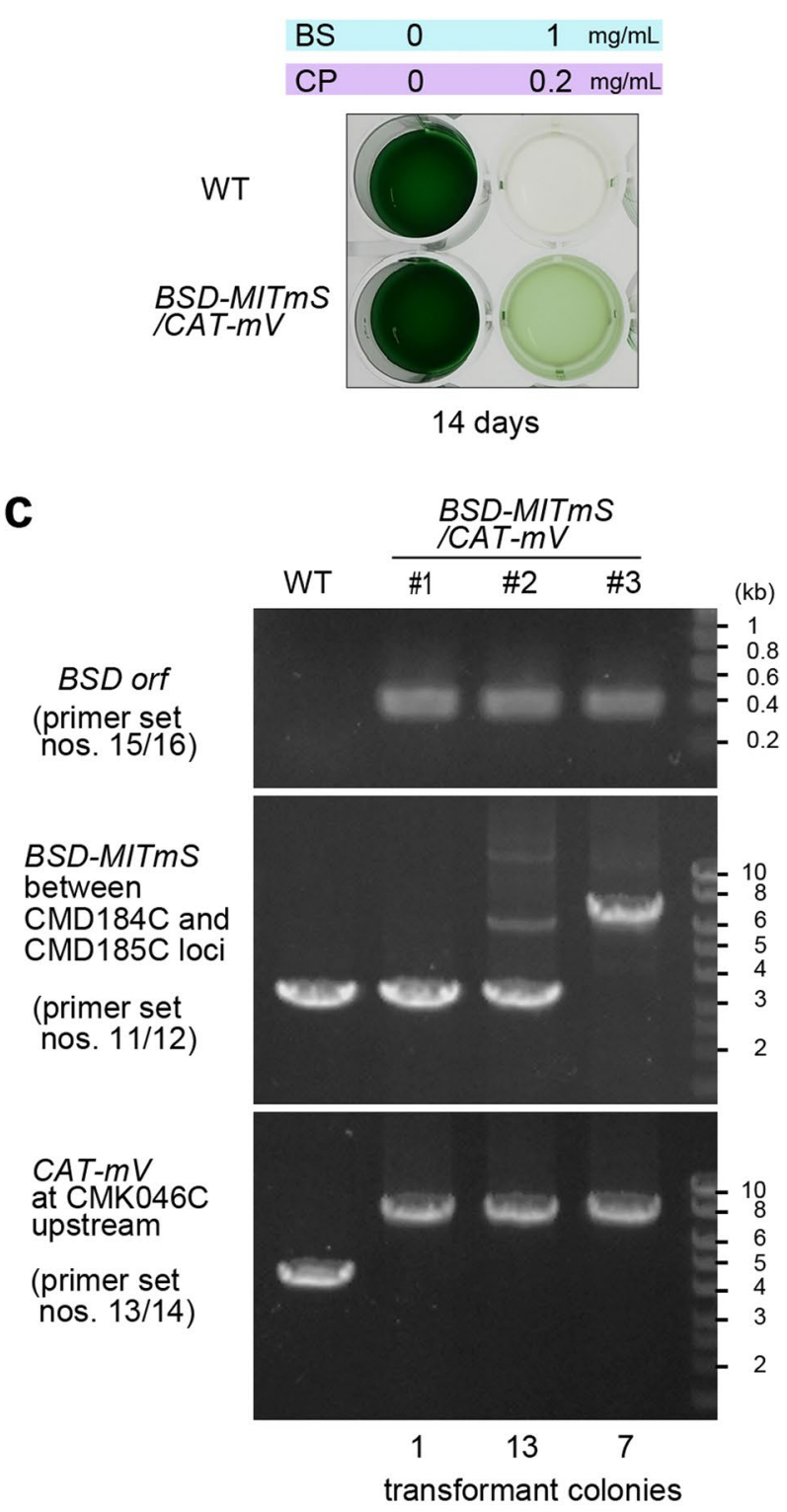

d


PC/mVENUS

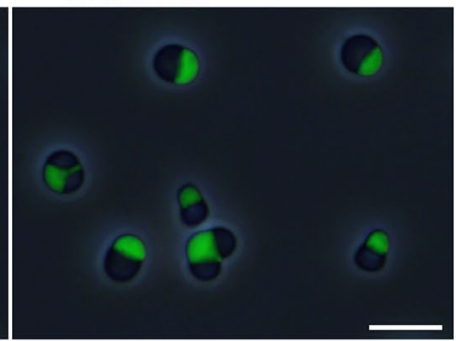

Fig. 3 (See legend on previous page.) 
emitted mVENUS fluorescence (Fig. 3d). These results demonstrated that cotransformation is feasible in $C$. merolae.

In the preset study, the efficiency of on-target insertion of the BSD-MITmS construct was lower than that of the CAT- $m V$ construct. Because the BSD marker was inserted into the genome by off-target insertion in some transformant clones, further optimization of the homology arms in the BSD-MITmSCARLET construct would be required to improve the targeting efficiency.

In the cotransformation system described above, it took 2 weeks to select BS and CP-resistant cells in a liquid medium after transformation and another 2 weeks to obtain single colonies of intended transformants on a gellan gum plate, which was faster than the previous procedures for a two-step sequential transformation [30, 31]. In addition, the newly developed $B S D$ selectable marker will enable an additional genetic modification to the already generated $C$. merolae transformants based on the $U R A$ or CAT system. Besides these advantages, a largesized DNA will likely be introduced into a chromosome by conjugating $B S D$ and $C A T$ markers to the respective borders of DNA. Multiple genetic modifications will also be useful for metabolic engineering because such modifications often require the inactivation of two or more enzymatic genes [47].

\section{Conclusions}

This study has developed a transformant selection method using BS and BSD in C. merolae. In addition, a cotransformation system has been developed to simultaneously modify two different chromosomal loci by a combination of $B S D$ and $C A T$ selectable markers. These methods and the simple nature of $C$. merolae cellular and genomic architecture will facilitate studies on several phenomena in photosynthetic eukaryotes.

\section{Methods}

\section{Algal culture}

C. merolae 10D WT (NIES-3377), BSD-MITmS, and $B S D-M I T m S / C A T-m V$ strains were maintained in MA2 liquid medium [2] [ $\left(\mathrm{NH}_{4}\right)_{2} \mathrm{SO}_{4} 40 \mathrm{mM}, \mathrm{KH}_{2} \mathrm{PO}_{4} 8 \mathrm{mM}$, $\mathrm{MgSO}_{4} 4 \mathrm{mM}, \mathrm{CaCl}_{2} 1 \mathrm{mM}, \mathrm{FeCl}_{3} 100 \mu \mathrm{M}$, EDTA-2Na $72 \mu \mathrm{M}, \mathrm{ZnCl}_{2} 2.8 \mu \mathrm{M}, \mathrm{MnCl}_{2} 16 \mu \mathrm{M}, \mathrm{Na}_{2} \mathrm{MoO}_{4} 7.2 \mu \mathrm{M}$, $\mathrm{CuCl}_{2} 1.3 \mu \mathrm{M}$, and $\mathrm{CoCl}_{2} 0.7 \mu \mathrm{M}$; the $\mathrm{pH}$ was adjusted to 2.3 with $\mathrm{H}_{2} \mathrm{SO}_{4}$ ] in $60 \mathrm{~mL}$ tissue culture flasks (TPP Techno Plastic Products AG), with agitation at $120 \mathrm{rpm}$ under continuous white light $\left(30 \mu \mathrm{mol} \cdot \mathrm{m}^{-2} \cdot \mathrm{s}^{-1}\right)$ at $42^{\circ} \mathrm{C}$.

\section{Preparation of linear DNA for C. merolae transformation}

The sequences and primers used in this study are listed in Supplementary Table S1. Linear DNA for the transformation of C. merolae was prepared as described below.
To generate the C. merolae BSD-MITmS strain, DNA that contained the BSD selectable marker and MITm$S C A R L E T$ expression cassette was prepared. For the homologous recombination at an intergenic region between CMD184C and CMD185C loci in the nuclear genome, the BSD selectable marker and MITmSCAR$L E T$ expression cassette were tandemly connected and sandwiched between sequences identical to CMD184C and CMDl85C loci, respectively (Fig. 1b), as described below. DNA fragment I [a portion of CMD184C orf (773rd nucleotide to the stop codon) flanked with the 25 bp downstream sequence] was amplified by PCR with the primer set \#1/2 using C. merolae WT genomic DNA as a template. DNA fragment II consisting of the $C A B$ (CMN234C) promoter, BSD orf, and APX (CMM158C) $3^{\prime}-u t r$ and DNA fragment III consisting of the $F B A$ (CMI049C) promoter, sequence encoding the mitochondrion-targeting peptide of EFTu (CMS502C), mVENUS orf (codon-optimized to $C$. merolae nuclear genome), and $\beta$-tubulin (CMN263C) 3'-utr were commercially synthesized. DNA fragment IV (sequence from the 28th to 1880th nucleotide downstream of CMD184C orf; this sequence corresponded to CMD185 and a portion of CMD186) was amplified by PCR with the primer set \#3/4 using C. merolae WT genomic DNA as a template. DNA fragments I to IV were assembled and cloned into the plasmid pUC19 vector (TAKARA, Japan) using the In-Fusion HD cloning kit (TAKARA) for amplification in Escherichia coli. The assembled DNA fragments I to IV were amplified by PCR with the primer set \#5/6 to prepare linear DNA for C. merolae transformation. The PCR product was purified using the QIAquick PCR Purification Kit (Qiagen).

To generate the C. merolae BSD-MITmS/CAT- $m V$, DNA containing the CAT selectable marker and $m V E$ $N U S$ expression cassette was prepared. For homologous recombination at an intergenic region at CMK046C upstream, the tandemly connected $C A T$ selectable marker and $m V E N U S$ expression cassette were sandwiched between two genomic sequences around the CMK046C locus as described below. DNA fragment $\mathrm{V}$ (a chromosomal sequence from 2300 to $898 \mathrm{bp}$ upstream of CMK046C orf) was amplified by PCR with the primer set $\# 7 / 8$ using $C$. merolae WT genomic DNA as a template. DNA fragment VI consisting of CPCC (CMP166C) promoter, $m$ VENUS orf, and ubiquitin (CMK296C) 3'-utr and DNA fragment VII consisting of $A P C C(C M O 250 C)$ promoter, sequence encoding the chloroplast-targeting peptide of APCC, CAT orf, and $\beta$-tubulin $3^{\prime}$-utr were commercially synthesized. DNA fragment VIII (CMK046C orf flanked with $897 \mathrm{bp}$ upstream and $471 \mathrm{bp}$ downstream sequences) was amplified by PCR with the primer set $\# 9 / 10$ using $C$. 
merolae WT genomic DNA as a template. DNA fragments V to VIII were assembled and cloned into the plasmid pUC19 vector using the In-Fusion HD cloning kit for amplification in E. coli. The assembled DNA fragments V to VIII were amplified by PCR with the primer set \#5/6 to prepare linear DNA for C. merolae transformation. The PCR product was purified using the QIAquick PCR Purification Kit.

\section{Transformation of C. merolae}

The transformation of $C$. merolae was carried out by the PEG-mediated method [46], as described previously $[2,30]$, with minor modifications. To generate the $B S D$ $M I T m S$ strain, $4 \mu \mathrm{g}$ of $B S D-M I T m S$ PCR product was introduced into $C$. merolae WT. To generate the $B S D$ $M I T m S / C A T-m V$ strain, $4 \mu \mathrm{g}$ each of BSD-MITmS and $C A T-m V$ PCR products were mixed $(8 \mu \mathrm{g}$ in total $)$ and introduced into $C$. merolae WT.

The transformed cells were cultured for 2 days in $8 \mathrm{~mL}$ of MA2 liquid medium in one well of a 6-well plate (VIOLAMO) in a $\mathrm{CO}_{2}(3 \%)$ incubator at $42^{\circ} \mathrm{C}$ in the light $(40 \mu \mathrm{E})$ for recovery and selected on an MA2 gellan gum plate supplemented with $1,1.25$, and $1.5 \mathrm{mg} / \mathrm{mL}$ BS (the preparation procedure is described below) or $2 \mathrm{~mL}$ MA2 liquid medium supplemented with both $1 \mathrm{mg} / \mathrm{mL}$ BS and $0.2 \mathrm{mg} / \mathrm{mL} \mathrm{CP}$ (the concentration was defined previously [30];) in one well of a 24-well plate (TPP Techno Plastic Products). Colony PCR analysis was carried out to verify targeted insertions of constructs into the intergenic regions between CMD184C and CMD185C loci and upstream of the CMK046C locus using the primer sets $\# 11 / 12$ and \#13/14, respectively. The BSD marker integration into the genome in transformants was verified by PCR using the primer set \#15/16.

\section{MA2 gellan gum plate supplemented with BS}

The MA2 gellan gum plate was prepared, as described previously [8], with minor modifications. One milliliter of $1 \%$ gellan gum (FUJFILM, Japan) solution (autoclaved to dissolve gellan gum in water) and $1 \mathrm{~mL}$ of $2 \times$ MA2 liquid medium were poured into one well of a 24-well plate. Immediately, a stock solution of BS (Cayman Chemical, USA; $50 \mathrm{mg} / \mathrm{mL}$ in distilled water) was added to the mixture at a final concentration of $1.0,1.25$, and $1.5 \mathrm{mg} / \mathrm{mL}$ and mixed. After gellan gum had solidified, $0.5 \mathrm{~mL}$ of $40 \%$ slurry of cornstarch, suspended in MA2 liquid medium with $1,1.25$, and $1.5 \mathrm{mg} / \mathrm{mL} \mathrm{BS}$, was placed on the solidified medium (Fig. 2a). After cornstarch had sedimented onto the gellan gum plate, excess suspension solution was removed, and cornstarch was dried up on a clean bench.

\section{Microscopy}

Images of cells were captured using a fluorescence microscope (BX51; Olympus) equipped with a three-charge-coupled device camera system (DP71; Olympus). To detect mVENUS and mSCARLET fluorescence, narrow-band filter sets (U-MNIBA3; Olympus and XF37; Omega, respectively) were used.

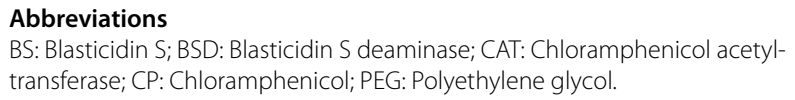

\section{Supplementary Information}

The online version contains supplementary material available at https://doi. org/10.1186/s12870-021-03365-z.

Additional file 1: Supplementary Figure S1. Full length unprocessed gel images of Figs. $2 \mathrm{c}$ and $3 \mathrm{c}$.

Additional file 2: Supplementary Table S1. List of nucleotide sequences of primers and synthetic DNA used in this study.

\section{Acknowledgments}

We thank Y. Yoshida (The University of Tokyo) for the information on fluorescent proteins and R. Ujigawa, R. Tomita, and U. Sugimoto for technical assistance in the Miyagishima laboratory.

\section{Authors' contributions}

Conceived and designed the experiments: TF, SH, and S-yM. Performed the experiments: TF and SH. Wrote the paper: TF and S-yM. The author(s) read and approved the final manuscript.

\section{Funding}

This work was supported by JSPS KAKENHI (grant no. 18 K06300 to TF, grant no. $20 \mathrm{~K} 06778$ to SH, and grant no. $20 \mathrm{H} 00477$ to S-yM), the JST-MIRAI Program of Japan Science and Technology Agency (to Sy-M), and the "Challenging Exploratory Research Projects for the Future" grant from the Research Organization of Information and Systems (to TF).

\section{Availability of data and materials}

The plasmids and C. merolae transformant strains generated in the current study are available from the corresponding authors upon request. The nucleotide sequences of C. merolae genetic loci (ID: CMN234C/CAB, CMM158C/APX, CMI049C/FBA, CMS502C/EFTu, CMN263C/ $\beta$-tubulin, CMP166C/CPCC,

CMO250C/APCC, CMD184C, CMD185C, CMD186C and CMK046C) used during the current study are available in Cyanidioschyzon merolae Genome Project v3 (http://czon.jp/).

\section{Declarations}

Ethics approval and consent to participate

No applicable.

\section{Consent for publication}

No applicable.

\section{Competing interests}

The authors declare there are no competing interests.

\section{Author details}

${ }^{1}$ Department of Gene Function and Phenomics, National Institute of Genetics, Mishima, Shizuoka 411-8540, Japan. ${ }^{2}$ Department of Genetics, Graduate University for Advanced Studies, SOKENDAI, Mishima, Shizuoka 411-8540, Japan. 
Received: 11 July 2021 Accepted: 24 November 2021

Published online: 04 December 2021

\section{References}

1. Miyagishima SY, Tanaka K. The unicellular red alga Cyanidioschyzon merolae - the simplest model of a photosynthetic eukaryote. Plant Cell Physiol. 2020(0):1-16. doi:https://doi.org/10.1093/pcp/pcab052.

2. Kuroiwa T, Miyagishima S, Matsunaga S, Sato N, Nozaki H, Tanaka K, Misumi M. Cyanidioschyzon merolae. A new model eukaryote for cell and organelle biology.. Springer Nature Singapore Pte Ltd. 2017:17-41. doi:https://doi.org/10.1007/978-981-10-6101-1

3. Matsuzaki M, Misumi O, Shin-I T, Maruyama S, Takahara M, Miyagishima SY, et al. Genome sequence of the ultrasmall unicellular red alga Cyanidioschyzon merolae 10D. Nature. 2004;428(6983):653-7. https://doi.org/10. 1038/nature02398.

4. Nozaki H, Takano H, Misumi O, Terasawa K, Matsuzaki M, Maruyama S, et al. A 100\%-complete sequence reveals unusually simple genomic features in the hot-spring red alga Cyanidioschyzon merolae. BMC Biol. 2007;5:28. https://doi.org/10.1186/1741-7007-5-28.

5. Suzuki K, Ehara T, Osafune T, Kuroiwa H, Kawano S, Kuroiwa T. Behavior of mitochondria, chloroplasts and their nuclei during the mitotic cycle in the ultramicroalga Cyanidioschyzon merolae. Eur J Cell Biol. 1994;63(2):280-8.

6. Imamura S, Terashita M, Ohnuma M, Maruyama S, Minoda A, et al. Nitrate assimilatory genes and their transcriptional regulation in a unicellular red alga Cyanidioschyzon merolae: genetic evidence for nitrite reduction by a sulfite reductase-like enzyme. Plant Cell Physiol. 2010;51(5):707-17. https://doi.org/10.1093/pcp/pcq043.

7. Fujiwara T, Tanaka K, Kuroiwa T, Hirano T. Spatiotemporal dynamics of condensins I and II: evolutionary insights from the primitive red alga Cyanidioschyzon merolae. Mol Biol Cell. 2013a;24(16):2515-27. https://doi. org/10.1091/mbc.E13-04-0208.

8. Fujiwara T, Ohnuma M, Yoshida M, Kuroiwa T, Hirano T. Gene targeting in the red alga Cyanidioschyzon merolae: single- and multi-copy insertion using authentic and chimeric selection markers. PLoS One. 2013b;8(9):e73608. https://doi.org/10.1371/journal.pone.0073608.

9. Sumiya N, Fujiwara T, Kobayashi Y, Misumi O, Miyagishima SY. Development of a heat-shock inducible gene expression system in the red alga Cyanidioschyzon merolae. PLoS One. 2014;9(10):e111261. https://doi.org/ 10.1371/journal.pone.0111261.

10. Fujiwara T, Kanesaki Y, Hirooka S, Era A, Sumiya N, Yoshikawa H, et al. A nitrogen source-dependent inducible and repressible gene expression system in the red alga Cyanidioschyzon merolae. Front Plant Sci. 2015;6:657. https://doi.org/10.3389/fpls.2015.00657.

11. Fujiwara T, Hirooka S, Ohbayashi R, Onuma R, Miyagishima SY. Relationship between cell cycle and diel transcriptomic changes in metabolism in a unicellular red alga. Plant Physiol. 2020;183(4):1484-501. https://doi. org/10.1104/pp.20.00469.

12. Kobayashi Y, Kanesaki Y, Tanaka A, Kuroiwa H, Kuroiwa T, Tanaka K. Tetrapyrrole signal as a cell-cycle coordinator from organelle to nuclear DNA replication in plant cells. Proc. Natl. Acad. Sci. U S A. 2009;106(3):803-7. https://doi.org/10.1073/pnas.0804270105.

13. Miyagishima SY, Nishida K, Mori T, Matsuzaki M, Higashiyama T, Kuroiwa $H$, et al. A plant-specific dynamin-related protein forms a ring at the chloroplast division site. Plant Cell. 2003;15(3):655-65. https://doi.org/10. 1105/tpc.009373.

14. Nishida K, Takahara M, Miyagishima SY, Kuroiwa H, Matsuzaki M, Kuroiwa T. Dynamic recruitment of dynamin for final mitochondrial severance in a primitive red alga. Proc. Natl. Acad. Sci. U S A. 2003;100(4):2146-51. https://doi.org/10.1073/pnas.0436886100.

15. Yoshida Y, Kuroiwa H, Misumi O, Yoshida M, Ohnuma M, Fujiwara T, et al. Chloroplasts divide by contraction of a bundle of nanofilaments consisting of polyglucan. Science. 2010;329(5994):949-53. https://doi.org/10. 1126/science.1190791.

16. Fujiwara T, Kuroiwa H, Yagisawa F, Ohnuma M, Yoshida Y, Yoshida M, et al. The coiled-coil protein VIG1 is essential for tethering vacuoles to mitochondria during vacuole inheritance of Cyanidioschyzon merolae. Plant Cell. 2010;22(3):772-81. https://doi.org/10.1105/tpc.109.070227.
17. Yagisawa F, Fujiwara T, Ohnuma M, Kuroiwa H, Nishida K, Imoto Y, et al. Golgi inheritance in the primitive red alga, Cyanidioschyzon merolae. Protoplasma. 2013;250(4):943-8. https://doi.org/10.1007/s00709-012-0467-6.

18. Imoto Y, Abe Y, Honsho M, Okumoto K, Ohnuma M, Kuroiwa H, et al. Onsite GTP fuelling via DYNAMO1 drives division of mitochondria and peroxisomes. Nat Commun. 2018;9(1):4634. https://doi.org/10.1038/ s41467-018-07009-z.

19. Miyagishima SY, Fujiwara T, Sumiya N, Hirooka S, Nakano A, Kabeya Y, et al. Translation-independent circadian control of the cell cycle in a unicellular photosynthetic eukaryote. Nat Commun. 2014;5:3807. https://doi.org/10. 1038/ncomms4807.

20. Imamura S, Kanesaki Y, Ohnuma M, Inouye T, Sekine Y, Fujiwara T, et al. R2R3-type MYB transcription factor, CmMYB1, is a central nitrogen assimilation regulator in Cyanidioschyzon merolae. Proc. Natl. Acad. Sci. U S A. 2009;106(30):12548-53. https://doi.org/10.1073/pnas.0902790106.

21. Moriyama T, Sakurai K, Sekine K, Sato N. Subcellular distribution of central carbohydrate metabolism pathways in the red alga Cyanidioschyzon merolae. Planta. 2014;240(3):585-98. https://doi.org/10.1007/ s00425-014-2108-0.

22. Takahashi S, Okubo R, Kanesaki Y, Zhou B, Takaya K, Watanabe S, et al. Identification of transcription factors and the regulatory genes involved in triacylglycerol accumulation in the unicellular red alga Cyanidioschyzon merolae. Plants (Basel). 2021;10(5):971. https://doi.org/10.3390/plants1005 0971.

23. KrupnikT, Kotabová E, van Bezouwen LS, Mazur R, Garstka M, Nixon PJ, et al. A reaction center-dependent photoprotection mechanism in a highly robust photosystem II from an extremophilic red alga, Cyanidioschyzon merolae. J Biol Chem. 2013;288(32):23529-42. https://doi.org/10. 1074/jbc.M113.484659.

24. Antoshvili M, Caspy I, Hippler M, Nelson N. Structure and function of photosystem I in Cyanidioschyzon merolae. Photosynth Res. 2019;139(13):499-508. https://doi.org/10.1007/s11120-018-0501-4.

25. Pi X, Tian L, Dai HE, Qin X, Cheng L, Kuang T, et al. Unique organization of photosystem I-light-harvesting supercomplex revealed by cryo-EM from a red alga. Proc. Natl. Acad. Sci U S A. 2018;115(17):4423-8. https://doi. org/10.1073/pnas.1722482115.

26. Haniewicz P, Abram M, Nosek L, Kirkpatrick J, El-Mohsnawy E, Olmos JDJ, et al. Molecular mechanisms of Photoadaptation of photosystem I Supercomplex from an evolutionary Cyanobacterial/algal intermediate. Plant Physiol. 2018;176(2):1433-51. https://doi.org/10.1104/pp.17.01022.

27. Stark MR, Dunn EA, Dunn WS, Grisdale CJ, Daniele AR, Halstead MR, et al. Dramatically reduced spliceosome in Cyanidioschyzon merolae. Proc. Natl. Acad. Sci U S A. 2015;112(11):E1191-200. https://doi.org/10.1073/pnas. 1416879112.

28. Mikulski P, Komarynets O, Fachinelli F, Weber APM, Schubert D. Characterization of the Polycomb-group mark H3K27me3 in unicellular algae. Front Plant Sci. 2017:8:607. https://doi.org/10.3389/fpls.2017.00607.

29. Zienkiewicz M, KrupnikT, Drożak A, Golke A, Romanowska E. Chloramphenicol acetyltransferase-a new selectable marker in stable nuclear transformation of the red alga Cyanidioschyzon merolae. Protoplasma. 2017;254(1):587-96. https://doi.org/10.1007/s00709-015-0936-9.

30. Fujiwara T, Ohnuma M, Kuroiwa T, Ohbayashi R, Hirooka S, Miyagishima SY. Development of a double nuclear gene-targeting method by twostep transformation based on a newly established chloramphenicolselection system in the red alga Cyanidioschyzon merolae. Front Plant Sci. 2017;8:343. https://doi.org/10.3389/fpls.2017.00343.

31. Takemura T, Imamura S, Kobayashi Y, Tanaka K. Construction of a selectable marker recycling system and the use in epitope tagging of multiple nuclear genes in the unicellular red alga Cyanidioschyzon merolae. Plant Cell Physiol. 2018;59(11):2308-16.

32. Minoda A, Sakagami R, Yagisawa F, Kuroiwa T, Tanaka K. Improvement of culture conditions and evidence for nuclear transformation by homologous recombination in a red alga, Cyanidioschyzon merolae 10D. Plant Cell Physiol. 2004;45(6):667-71. https://doi.org/10.1093/pcp/pch087.

33. Taki K, Sone T, Kobayashi Y, Watanabe S, Imamura S, Tanaka K. Construction of a URA5.3 deletion strain of the unicellular red alga Cyanidioschyzon merolae: A backgroundless host strain for transformation experiments J. Gen. Appl. Microbiol. 2015;61(5):211-214. doi: https://doi.org/10.2323/ jgam.61.211.

34. Takeuchi S, Hirayama F, Uera F, Sakai H, Yonehara H. Blasticidin S, a new antibiotic. J Antibiot (Tokyo) 1958;11(1):1-5. 
35. Svidritskiy E, Ling C, Ermolenko DN, Korostelev A. A Blasticidin S inhibits translation by trapping deformed tRNA on the ribosome Proc Natl Acad Sci USA 1993;110(30):12283-12288. doi: https://doi.org/10.1073/pnas. 1304922110.

36. Powers KT, Stevenson-Jones F, Yadav SKN, Amthor B, Bufton JC, Borucu $\mathrm{U}$, et al. Blasticidin $\mathrm{S}$ inhibits mammalian translation and enhances production of protein encoded by nonsense mRNA. Nucleic Acids Res. 2021;49(13):7665-79. https://doi.org/10.1093/nar/gkab532.

37. Iyer $L M$, Zhang D, Rogozin IB, Aravind L. Evolution of the deaminase fold and multiple origins of eukaryotic editing and mutagenic nucleic acid deaminases from bacterial toxin systems. Nucleic Acids Res. 2011;39(22):9473-97. https://doi.org/10.1093/nar/gkr691.

38. Seto $\mathrm{H}$, Ôtake $\mathrm{N}$, Yonehara H. Biological transformation of Blasticidin $\mathrm{S}$ by Aspergillus fumigatus sp. Agric Biol Chem. 1966;30(9):877-86. https://doi. org/10.1080/00021369.1966.10858693.

39. Endo T, Furuta K, Kaneko A, Katsuki T, Kobayashi K, Azuma A, Watanabe A, Shimazu A. Inactivation of blasticidin S by Bacillus cereus. I. inactivation mechanism. J. Antibiot. (Tokyo). 1987;40(12):1791-1793. doi: https://doi. org/10.7164/antibiotics.40.1791

40. Kamakura T, Yoneyama K, Yamaguchi I. Expression of the blasticidin S deaminase gene (bsr) in tobacco: fungicide tolerance and a new selective marker for transgenic plants. Mol Gen Genet. 1990;223(2):332-4. https://doi.org/10.1007/bf00265072.

41. Kimura M, Kamakura T, Tao QZ, Kaneko I, Yamaguchi I. Cloning of the blasticidin S deaminase gene (BSD) from Aspergillus terreus and its use as a selectable marker for Schizosaccharomyces pombe and Pyricularia oryzae. Mol Gen Genet. 1994;242(2):121-9. https://doi.org/10.1007/BF00391004.

42. Izumi M, Miyazawa H, Kamakura T, Yamaguchi I, Endo T, Hanaoka F. Blasticidin S-resistance gene (bsr): a novel selectable marker for mammalian cells. Exp Cell Res. 1991;197(2):229-33. https://doi.org/10.1016/00144827(91)90427-V.

43. Fukuda H, Kizaki Y. A new transformation system of Saccharomyces cerevisiae with blasticidin S deaminase gene. Biotechnol Lett. 1999;21:969-71. https://doi.org/10.1023/A:1005613206138.

44. Félix de Carpentier, Jeanne Le Peillet, Nicolas D. Boisset, Pierre Crozet, Stéphane D. Lemaire, and Antoine Danon. Blasticidin S Deaminase: A New Efficient Selectable Marker for Chlamydomonas reinhardtii. Front Plant Sci. 2020;11:242. doi: https://doi.org/10.3389/fpls.2020.00242

45. Imoto Y, Kuroiwa H, Yoshida Y, Ohnuma M, Fujiwara T, Yoshida M, et al. Single-membrane-bounded peroxisome division revealed by isolation of dynamin-based machinery. Proc. Natl. Acad. Sci. U S A. 2013:110(23):9583-8. https://doi.org/10.1073/pnas.1303483110.

46. Ohnuma M, Yokoyama T, Inouye T, Sekine Y, Tanaka K. Polyethylene glycol (PEG)-mediated transient gene expression in a red alga, Cyanidioschyzon merolae 10D. Plant Cell Physiol. 2008;49(1):117-20. https://doi.org/10. 1093/pcp/pcm157.

47. Alper H, Jin YS, Moxley JF, Stephanopoulos G. Identifying gene targets for the metabolic engineering of lycopene biosynthesis in Escherichia coli. Metab Eng. 2005;7(3):155-64. https://doi.org/10.1016/j.ymben.2004.12. 003.

\section{Publisher's Note}

Springer Nature remains neutral with regard to jurisdictional claims in published maps and institutional affiliations.

Ready to submit your research? Choose BMC and benefit from:

- fast, convenient online submission

- thorough peer review by experienced researchers in your field

- rapid publication on acceptance

- support for research data, including large and complex data types

- gold Open Access which fosters wider collaboration and increased citations

- maximum visibility for your research: over $100 \mathrm{M}$ website views per year

At BMC, research is always in progress.

Learn more biomedcentral.com/submissions 